\title{
A Rapid-ACCE review of CYP2C9 and VKORC1 alleles testing to inform warfarin dosing in adults at elevated risk for thrombotic events to avoid serious bleeding
}

\author{
Monica R. McClain, PhD ${ }^{1}$, Glenn E. Palomaki, BS ${ }^{1}$, Margaret Piper, PhD' ${ }^{2}$, and James E. Haddow, MD ${ }^{1}$
}

\begin{abstract}
Purpose: Summarize evidence regarding genetic testing in adults to inform warfarin dosing to reduce adverse drug events such as serious bleeding. Methods: Review published (and selected gray) literature using the Rapid-ACCE structure that addresses analytic validity, clinical validity, clinical utility, and ethical, legal, and social implications. Results: Preliminary data suggest overall analytic sensitivity and specificity will be $98 \%$ or higher for CYP2C9 genotyping, but strength of evidence for analytic validity is low, especially for VKORC1 testing. Strength of evidence is high for the clinical validity of both genes in predicting stable warfarin dose, an intermediate outcome, but is low for the association between CYP2C9 testing and severe bleeding events (clinical sensitivity $46 \%$ (95\% Cl 32-60\%); specificity $69 \%$ (95\% Cl 62-75\%) and absent for bleeding events associated with VKORC1 testing. No data are available to document clinical utility of genotyping before warfarin dosing. Conclusions: The most important gaps identified are: which variants should be included in a testing panel, lack of data from external proficiency testing, lack of validated dosing algorithm incorporating genetic and nongenetic factors, evidence of clinical utility, reliable economic analyses, and methods to address several ethical, legal, and social implications issues. Genet Med
\end{abstract} 2008:10(2):89-98.

Key Words: evidence review, warfarin, CYP2C9, VKORC1, severe bleeding

The main aim of this rapid-ACCE (Analytic validity, Clinical validity, Clinical utility, and Ethical, legal, and social implications) review ${ }^{1}$ is to systematically collect and evaluate the evidence regarding the efficacy of identifying cytochrome P-450 2C9 (CYP2C9) and vitamin K epoxide reductase complex 1 (VKORC1) alleles to guide warfarin dosing on the basis of this information, as a way to prevent occurrences of severe bleeding. The clinical scenario focuses on adult candidates for warfarin treatment, as a result of being at high risk for future thrombotic events. The clinical disorder(s) under consideration is a severe bleeding episode associated with warfarin treatment, such as hemorrhagic stroke, of sufficient severity to produce serious morbidity and mortality. Hemorrhagic events are a complication of warfarin drug treatment, because of the narrow therapeutic range. Thrombotic events are also a consequence of the narrow therapeutic range, but this evidence re-

\footnotetext{
From the ${ }^{1}$ Department of Pathology and Laboratory Medicine, Women and Infants Hospital, Warren Alpert School of Medicine of Brown University, Providence, Rhode Island; and ${ }^{2}$ Blue Cross and Blue Shield Technology Evaluation Center, Chicago, Illinois.

Monica R. McClain, PhD, Division of Medical Screening, Women and Infants Hospital, 70 Elm Street, 2nd Floor, Providence, RI 02903. E-mail: mmcclain@ipmms.org.

Disclosure: The authors declare no conflict of interest.

Submitted for publication May 18, 2007.

Accepted for publication September 4, 2007.

DOI: 10.1097/GIM.0b013e31815bf924
}

view was limited to the hemorrhagic events. The target range for monitoring warfarin therapy is an International Normalized Ratio (INR) value between 2.0 and 3.0 (slightly lower or higher for some conditions), which is a standardized measure of the patient's prothrombin time, such that results are comparable across laboratories and test reagents. ${ }^{2}$ The risk for serious bleeding increases when INR values are 4.0 or higher, and such elevations are more likely to occur within the first few weeks after initiating warfarin treatment, before a stable dose and INR are achieved. It is likely that maintenance of warfarin doses will continue to be primarily based on INR measurements, but genotyping may be of help with initial dosing and obtaining stable INR more quickly.

The objectives of the present review are to: (1) briefly evaluate and summarize existing knowledge, (2) provide information to aid in developing clinical and laboratory guidelines for CYP2C9 and VKORC1 alleles testing to guide warfarin dosing, (3) provide information to be used in developing patient/physician education materials, and (4) identify gaps in knowledge from which a research agenda can be developed. Understanding the extent of benefit to be gained by testing is important, because: (1) a large number of new warfarin patients per year might have genetic tests performed (hundreds of thousands to as many as 2 million), (2) up to 800 reportable adverse drug events associated with warfarin usage per year occur in the United States, ${ }^{3}$ (3) the Food and Drug Administration has re- 
cently revised the Coumadin ${ }^{\circledR}$ label (and will revise the generic warfarin label) to include genomic test information without mandating genetic testing, and (4) CYP2C9/VKORC1 testing services may soon be readily available. The complete evidence report is available at http://www.acmg.net.

\section{MATERIALS AND METHODS}

The ACCE methodology is specifically designed to facilitate the appropriate transition of genetic tests from investigational settings to clinical and public health practice. ${ }^{1}$ The 44 -question format was developed as part of a Centers for Disease Control and Prevention-sponsored project (CCU119356-01) to assess the availability, quality, and usefulness of existing data on DNA-based tests and testing algorithms. This methodology is both time consuming and expensive, with a full review taking many months to complete, at a cost of $\$ 50,000-\$ 100,000$, or more. In an effort to provide an evidence-based review in a more timely fashion, the rapid-ACCE methodology was recently developed. ${ }^{4}$ Examples of when this methodology is appropriate include: topic areas with a small evidence base and topics that are narrowly defined (e.g., stakeholders require a review of only a limited set of ACCE questions or a review for a specific population or assay methodology). Depending on the amount of literature and availability of experts to aid the interpretation of complex issues, rapid-ACCE reviews might be completed for between $\$ 10,000$ and $\$ 40,000$ within a few months. This review utilizes the rapid-ACCE approach.

Before assessment begins, ACCE requires that the disorder, clinical scenario, and test(s) be clearly defined and agreed upon by all stakeholders. For this review, the stakeholder was an American College of Medical Genetics panel whose charge was to issue a recommendation for CYP2C9/VKORC1 pharmacogenetic testing for warfarin/Coumadin administration (page 139, this issue). ${ }^{5}$ This panel was composed of 14 experts in the area of pharmacology, molecular genetics, clinical genetics, bioethics, economics, and anticoagulation. The ACCE questions are divided into four major sections, defined as follows. Analytic validity refers to the ability of laboratory testing to correctly identify the genotypes of interest (analytic sensitivity and specificity) in the analytic, as well as preanalytic and postanalytic phases. Other areas included are robustness, repeatability, and quality control. Clinical validity refers to the test's ability to correctly identify the phenotype of interest. For example, clinical sensitivity for CYP2C9 would be defined as the proportion of all severe bleeding events that occurred among nonwild genotypes. Clinical validity would also include the positive and negative predictive values (PPV and NPV), genotype/phenotype associations and penetrance. Clinical utility assesses risks and benefits of testing. For a test to have clinical utility, the results must be used to impact some aspect of patient care which leads to a measurable improvement in outcome that matters to the patient. Clinical utility also can include information regarding implementation issues such as pilot trials, needed resources, validated educational materials, and economic implications. Some of the ethical, legal, and so- cial implications (ELSI) are included in other sections (e.g., informed consent, addressed as part of clinical utility). Other topics, such as discrimination, health disparities, patents, obligation to disclose, and the existence of effective safeguards, are addressed under this section.

Data used in each of these sections were obtained by literature searches (e.g., PubMed) and reference lists from retrieved articles. Meta-analyses were particularly sought as a way to identify existing analyses and methodology. When published data were not available, or did not cover the question adequately, data were sought via the gray literature. This included FDA submissions, laboratory web-site information, abstracts, and materials distributed at meetings. In some instances, individuals who likely held the relevant information were directly contacted and asked to collaborate. Data from the gray literature were labeled as such to avoid confusion with published literature.

Each study was evaluated for the strength of the study design (randomized trial being the highest), sample size, avoidance/identification of biases, description of population, and comparison to a gold standard. The ratings are as follows: marginal-multiple deficiencies that cast doubt on the conclusions, gray literature; adequate- deficiencies identified, but conclusions likely to be reliable; and good-few, if any deficiencies in study evaluation. The strength of evidence combines the available studies with formal (or informal) tests of heterogeneity of effect, with the following ratings: low-one, or several marginal to adequate studies with heterogeneity; medium - multiple adequate studies (or multiple studies with at least one good study) with homogeneity, or multiple good studies with heterogeneity; and high — multiple good studies with homogeneity. Strength of effect is independent of strength of evidence. For example, there can be a high strength of evidence rating for a weak measure of effect (e.g., confident that the odds ratio is 1.3).

\section{RESULTS}

\section{Analytic validity}

The cytochrome P450 complex is a group of hepatic microsomal enzymes responsible for the oxidative metabolism of various substrates (pharmacokinetics). Thirty-seven CYP2C9 haplotypes containing over 100 variants have been identified, but the literature focuses on two of these that are associated with reduced metabolism of warfarin. These are designated as ${ }^{\star} 2(\mathrm{R} 144 \mathrm{C}, 3608 \mathrm{C}>\mathrm{T})$ and ${ }^{\star} 3$ (I359L, $\left.42614 \mathrm{~A}>\mathrm{C}\right)$ variants. ${ }^{\star} 1$ is the designation for the wild-type allele. The frequencies of the $\star^{\star}$ and ${ }^{*} 3$ variants are approximately $12.2 \%$ and $7.9 \%$, respectively, in the European Caucasian population. ${ }^{6}$ Individuals with the wild genotype reach a warfarin steady state in 3-5 days. Heterozygotes for ${ }^{\star} 2$ and ${ }^{\star} 3$ require $6-8$ days and $12-15$ days, respectively. ${ }^{7}$ Three additional variants $\left({ }^{\star} 4\right.$ or I359T or $42615 \mathrm{~T}>\mathrm{C}$ [Ile359Thr]; ${ }^{\star} 5$ or D360E or $42619 \mathrm{C}>\mathrm{G}$ [Asp360Alu]; and ${ }^{\star} 6$ or 10601 delA or $818 \mathrm{delA}$ ) are sometimes mentioned for inclusion in a testing panel for African Americans or Asian Americans. However, even in these populations, the allele frequencies for ${ }^{\star} 4,{ }^{\star} 5$, and ${ }^{\star} 6$ are $<1 \%{ }^{8}$ Table 1 
Table 1

CYP2C9 variants and their relationship to warfarin metabolism and a VKORC1 variant and its relationship to gene expression

\begin{tabular}{|c|c|c|}
\hline \multicolumn{3}{|c|}{ CYP2C9 } \\
\hline Genotype & Metabolism & Nomenclature \\
\hline${ }^{\star} 1 /{ }^{\star} 1$ & Extensive, rapid, ultra-metabolizer & Normal, wild \\
\hline${ }^{\star} 1 /{ }^{\star} 2$ & Intermediate & Heterozygote \\
\hline${ }^{\star} 1 /{ }^{*}$ & Poor, slow & Heterozygote \\
\hline${ }^{\star} 2 /{ }^{*} 3$ & Poor, slow & Compound heterozygote \\
\hline${ }^{\star} 2 / * 2$ & Poor, slow & Homozygote \\
\hline$\star 3 / * 3$ & Extremely slow & Homozygote \\
\hline \multicolumn{3}{|c|}{ VKORC1 } \\
\hline Genotype & Enzyme production & Nomenclature \\
\hline BB & Low (higher warfarin dose) & Normal, wild \\
\hline $\mathrm{AB}$ & Medium & Heterozygote \\
\hline AA & High (lower warfarin dose) & Homozygote \\
\hline
\end{tabular}

shows the most common CYP2C9 genotypes, their associated warfarin metabolic rates and nomenclature.

Variants in the gene encoding VKORC1 have also been determined to affect the response to warfarin via reduced enzyme activity (pharmacodynamics). The clinically relevant variants $(-1639 \mathrm{G}>\mathrm{A}, 1173 \mathrm{C}>\mathrm{T}, 1542 \mathrm{G}>\mathrm{C}, 2255 \mathrm{~T}>\mathrm{C}, 3730 \mathrm{G}>\mathrm{A})$ in
non-Hispanic Caucasians are in strong linkage disequilibrium. There are several conflicting nomenclatures used to refer to these variants. We have chosen to use the nomenclature by Rieder et al. ${ }^{9}$ Table 1 shows the relationship between VKORC1 genotype and warfarin dose. The frequencies of these genotypes have been estimated from data reported by several studies, ${ }^{10-13}$ using a random effects model. Among non-Hispanic Caucasians, these frequencies are $35 \%, 47 \%$, and $18 \%$ for the $\mathrm{BB}, \mathrm{AB}$, and $\mathrm{AA}$ genotypes, respectively. Other studies have reported wide variation of these frequencies by race/ethnicity (Question 22). ${ }^{8,9,14-18}$ While VKORC1 variants are considerably more prevalent than those of CYP2C9, there are fewer data available that characterize their analytic validity and clinical validity.

Nearly all available data for analytic validity refer to the detection of two variants in the CYP2C9 gene; few data are available about detecting the variants in the VKORC1 gene. Based on seven studies reporting performance in the analytic phase of testing (Table 2), assays for the common CYP2C9 genotypes $\left({ }^{\star} 1 /{ }^{\star} 2\right.$ and $\left.{ }^{\star} 1 /{ }^{\star} 3\right)$ have an analytic sensitivity of $100 \%$ (95\% CI $96.7-100 \%) .{ }^{19-25}$ The analytic specificity is also $100 \%$ (95\% CI 98.2-100\%). Based on sparse data for the less common CYP2C9 genotypes $\left({ }^{\star} 2 /{ }^{\star} 2,{ }^{\star} 2 /{ }^{\star} 3\right.$, and $\left.{ }^{\star} 3 /{ }^{\star} 3\right)$ the analytic sensitivity of selected assay systems is still $100 \%$, but the confidence interval is wider (95\% CI 75-100\%).20,22,23,25 The bottom of Table 2 also contains information from the gray literature regarding both CYP2C9 and VKORC1 testing. No published information is available to directly estimate preana-

Table 2

Analytic validity of CYP2C9 (restricted to the ${ }^{\star} 2$ and ${ }^{\star} 3$ variants) and $V K O R C 1$ testing

\begin{tabular}{|c|c|c|c|c|c|c|c|c|c|}
\hline \multirow[b]{2}{*}{ Reference } & \multirow[b]{2}{*}{ Year } & \multirow[b]{2}{*}{ Assay method } & \multirow[b]{2}{*}{ Referent method } & \multicolumn{5}{|c|}{$\begin{array}{c}\text { CYP2C9 } \\
\text { Analytic sensitivity (test result/referent result) }\end{array}$} & \multirow{2}{*}{$\begin{array}{c}\text { Analytic } \\
\text { specificity } \\
\left({ }^{\star} 1,{ }^{\star} 1\right)\end{array}$} \\
\hline & & & & $\left({ }^{\star} 1,{ }^{\star} 2\right)$ & $\left({ }^{\star} 2,{ }^{\star} 2\right)$ & $\left({ }^{\star} 1,{ }^{\star} 3\right)$ & $\left({ }^{\star} 3,{ }^{\star} 3\right)$ & $\left({ }^{\star} 2,{ }^{\star} 3\right)$ & \\
\hline Hillman et al. ${ }^{22}$ & 2004 & LightCycler & Sequencing & $2 / 2$ & $1 / 1$ & - & $1 / 1$ & $1 / 1$ & $4 / 4$ \\
\hline Pickering et al. ${ }^{23}$ & 2004 & Luminex, eSensor & Sequencing & $15 / 15$ & $1 / 1$ & $13 / 13$ & - & $2 / 2$ & $70 / 70$ \\
\hline Wen et al. ${ }^{24}$ & 2003 & Microarray & Sequencing & - & - & $7 / 7$ & - & - & $13 / 13$ \\
\hline Zainuddin et al. ${ }^{25}$ & 2003 & Nested PCR & Sequencing & $3 / 3$ & - & $5 / 5$ & $2 / 2$ & $2 / 2$ & $28 / 28$ \\
\hline Eriksson et al. ${ }^{21}$ & 2002 & Pyrosequencing & PCR-RFLP & $9 / 9$ & - & $5 / 5$ & - & - & $9 / 9$ \\
\hline Aquilante et al. ${ }^{19}$ & 2004 & Pyrosequencing & PCR-RFLP & - & - & - & - & - & - \\
\hline Burian et al. ${ }^{20}$ & 2002 & LightCycler & PCR-RFLP & $27 / 27$ & $1 / 1$ & $10 / 10$ & $1 / 1$ & $1 / 1$ & $79 / 79$ \\
\hline Total & & & & $56 / 56$ & $3 / 3$ & $40 / 40$ & $4 / 4$ & $6 / 6$ & $203 / 203$ \\
\hline Third Wave Tech & 2006 & Invader, Tag-It, Pyro & Sequencing & $9 / 9$ & $3 / 3$ & $6 / 6$ & $2 / 2$ & $6 / 6$ & $9 / 9$ \\
\hline ARUP Laboratory & 2006 & Invader, Tag-It & Sequencing & $9 / 9$ & - & $1 / 1$ & - & - & $21 / 21$ \\
\hline \multirow[t]{3}{*}{ LabCorp } & 2006 & Invader, Tag-It & PCR-RFLP & $6 / 6$ & $1 / 1$ & $5 / 5$ & $1 / 1$ & $4 / 4$ & $5 / 5$ \\
\hline & & & & \multicolumn{5}{|c|}{ VKORC1 } & \\
\hline & & & & $\mathrm{AB}$ & AA & & & & $\mathrm{BB}$ \\
\hline Third Wave Tech & 2006 & Invader, Pyro & Sequencing & $16 / 16$ & $12 / 12$ & & & & $7 / 7$ \\
\hline ARUP & 2006 & Invader & Sequencing & $10 / 10$ & $4 / 4$ & & & & $17 / 17$ \\
\hline LabCorp & 2006 & Invader & PCR-RFLP, sequencing & $10 / 10$ & $5 / 5$ & & & & $7 / 7$ \\
\hline
\end{tabular}

ARUP, Associated Regional and University Pathologists. 
Table 3

Relative risk of INR values above 3.0 during warfarin induction, stratified by CYP2C9 genotype

\begin{tabular}{lccc}
\hline & Week after induction & Lindh et al., $2005^{32}$ & Peyvandi et al., 2004 \\
\hline Relative risk $\left({ }^{*} 2\right.$ vs. $\left.{ }^{*} 1 /{ }^{*} 1\right)$ & 1 & $2.8(1.2-6.7)^{a}$ & $1.9(1.3-2.3)$ \\
& 2 & $2.1(1.2-3.7)$ & $1.8(1.3-2.3)$ \\
Relative risk $\left({ }^{*} 3\right.$ vs. $\left.{ }^{*} 1 /{ }^{*} 1\right)$ & 3 & $1.0(0.5-1.8)$ & $2.0(1.3-3.1)$ \\
& 1 & $3.4(2.5-12)$ & $2.5(1.3-4.5)$ \\
\hline
\end{tabular}

${ }^{\star} 2$ includes ${ }^{*} 1 /{ }^{*} 2$ and ${ }^{\star} 2 /{ }^{*} 2 ;{ }^{*} 3$ includes ${ }^{\star} 1 /{ }^{*} 3,{ }^{*} 3 /{ }^{*} 3$, and ${ }^{\star} 2 /{ }^{*} 3$.

${ }^{a} 95 \%$ confidence interval.

lytic or postanalytic errors. Depending on the methodology, sample type and sample condition, $1-5 \%$ of samples may experience repeated assay failures resulting in inconclusive test results $^{19}$ (see Question 16 in the full review for personal communications containing additional data). These failures can be viewed as reducing the analytic sensitivity and specificity.

Based on other molecular tests that have been studied in more detail (cystic fibrosis gene ${ }^{26}$ and hereditary hemochromatosis gene ${ }^{27,28}$ ), working estimates of overall analytic sensitivity and specificity for the common CYP2C9 genotypes are $98-99 \%$ and $99.5-99.75 \%$, respectively. Too few data exist to estimate these rates for VKORC1 genotyping. Nearly all available data are based on DNA extracted from whole blood samples. Other sample types (e.g., mouthwash) have been mentioned, ${ }^{29}$ but data are sparse. Using these estimates for CYP2C9, incorrect genotype assignments would be expected to be relatively rare (1 in 50 to 1 in 200) among any genotype group. At least 12 laboratories in the United States now offer CYP2C9 and/or VKORC1 genotyping for clinical use (see full report, Table 3). Several manufacturers offer reagents to test for variants in both genes.

It appears that the methodologies used to identify CYP2C9 and $V K O R C 1$ variants can easily be completed in a day. Thus, turn-around-time $>2$ or 3 days will be because of slow transport of samples, or that the laboratory does not run the assay every day. Neither of these issues would be expected to impact analytic validity (other than to perhaps improve the quality of samples by shortening transport time). On at least one website offering testing, the laboratory turn-around-time is stated to be 1 day (http://www.kimballgenetics.com/tests.html).

The Genetic Testing Quality Control Materials Program at the CDC assists genetic testing laboratories in obtaining validated quality control materials. As part of this program, 96 samples from Coriell Cell Repositories (Camden, NJ) were genotyped for CYP2C9 and VKORC1 variants (www.phppo. cdc.gov/dls/genetics/qcmaterials/pdf/CYP2C9_VKORC1.pdf). Two laboratories used the Tag-It (TM Bioscience) methodology to analyze the CYP2C9 gene, and both identified the same genotypes in all samples. Two other laboratories sequenced the VKORC1 gene, and both identified the same genotypes in all samples. Laboratories validating new assays can purchase these samples with known genotypes.
The College of American Pathologists has established a working group consisting of members from the College of American Pathologists/American College of Medical Genetics, Biochemical and Molecular Genetics, Special Chemistry, Toxicology, and Coagulation Committees, to develop a Pharmacogenomics (PGx) Survey for 2007. This PGx Survey will ship twice per year (April and September). Each shipment will contain two different vials of $25 \mu \mathrm{g}$ each of extracted DNA, which participants will be able to test for genetic variations in the CYP2C19, CYP2C9, CYP2D6, UGT1A1, and VKORC1 genes (see www.cap.org for updates).

Gaps in knowledge include: (1) which CYP2C9/VKORC1 variants should be part of a clinical panel, (2) poorly defined analytic validity for the less common CYP2C9 genotype (e.g., ${ }^{\star} 3 /{ }^{*} 3$ ), (3) published data on analytic validity for VKORC1 against a "gold standard," (4) whether clinical laboratories are able to offer an appropriately validated test (e.g., variants included, turn-around time, costs, sample types, internal analytic validity studies), (5) limited information on long-term performance/consistency of methods (within-laboratory variability), (6) data showing between-laboratory consistency, (7) overall estimate of analytic performance including preanalytic and postanalytic error rates, (8) method-specific and samplespecific failure rates, and (9) data from the external proficiency testing program.

\section{Clinical validity}

Clinical validity was examined using one intermediate outcome (elevated INRs), as well as the health outcome of severe bleeding. INR values above 3.0 are twice as likely among CYP2C9 heterozygotes (relative risk of 2.0 or higher), and are more likely to occur in the first and second week (induction phase) after warfarin initiation than in the third week or later (Table 3). This information is based on only two studies that were designed and analyzed differently. ${ }^{34,35} \mathrm{~A}$ third study found a weak correlation between the rate of change in the INR values (slope) and CYP2C9 genotype (nonwild genotypes had a higher slope, $P=0.05){ }^{36}$

Clinical sensitivity is defined as the proportion of individuals with the outcome of interest (severe bleeding) that have a genotype other than wild (i.e., ${ }^{\star} 1 /{ }^{\star} 2,{ }^{\star} 2 /{ }^{\star} 2,{ }^{\star} 2 /{ }^{\star} 3,{ }^{\star} 1 /{ }^{\star} 3,{ }^{\star} 3 /$ $\left.{ }^{\star} 3\right)$. This is synonymous with the detection rate. With nonwild 
Table 4

Clinical sensitivity, clinical specificity, relative risk, and attributable risk for severe bleeding events (wild vs. nonwild CYP2C9 genotype)

\begin{tabular}{|c|c|c|c|c|}
\hline Study & Clinical sensitivity (\%) & Clinical specificity (\%) & Relative risk (\%) & Attributable risk (\%) \\
\hline Ogg et al. ${ }^{30 a}$ & 23 & 87 & 1.85 & 7 \\
\hline Margaglione et al. ${ }^{31 b}$ & 67 & 53 & 1.91 & 12 \\
\hline Higashi et al. ${ }^{32}$ & 50 & 72 & 2.19 & 15 \\
\hline Wadelius et al. ${ }^{33}$ & 33 & 66 & 0.96 & 0 \\
\hline Summary of Higashi and Wadelius (95\% CI) & $46(32-60)$ & $69(62-75)$ & $1.7(0.8-3.6)$ & $7(0-15)$ \\
\hline
\end{tabular}

${ }^{a}$ Considered only ${ }^{*} 3$ genotypes (these estimates are not included in the summary line).

${ }^{b}$ Wild CYP2C9 genotype frequency in Italy is low (these estimates are not included in the summary line).

CYP2C9 genotypes grouped together from two studies, the clinical sensitivity of CYP2C9 to identify serious bleeding events is $46 \%$ (95\% CI 32-60\%), ${ }^{32,33}$ indicating that about half of all serious bleeding events occur among CYP2C9 wild-type individuals (Table 4). Clinical specificity is defined as the proportion of individuals with no severe bleeding that have the wild $\left({ }^{*} 1 /{ }^{*} 1\right)$ genotype. One minus the clinical specificity is the false positive rate. The false positive rate indicates the proportion of individuals without a bleeding event that have a nonwild genotype. Overall, the clinical specificity of CYP2C9 is $69 \%$ (95\% CI 62-75\%). The correspondingly high false positive rate $(31 \%)$ is because nonwild CYP2C9 genotypes are relatively common and most will not experience serious bleeding.
The relative risk for serious bleeding in nonwild versus wild individuals is 1.7 (95\% CI $0.8-3.6)$, consistent with an occasional warfarin overdose in the presence of a nonwild genotype.

Figure 1 shows the relationship between these parameters in a population with a serious bleeding rate of $5 \%$. The prevalence of serious bleeding among populations varies widely $(<1-17 \%)$ depending on many factors ${ }^{37-46}$ (e.g., indication for warfarin, age, comorbidities, definition of serious bleeding, and other drug use). The PPV is estimated to be 7\% (i.e., 1 in 14 patients with a nonwild CYP2C9 genotype will suffer a bleeding event). Because nonwild CYP2C9 genotypes are relatively common and the prevalence of serious bleeding is low, most will not experi-

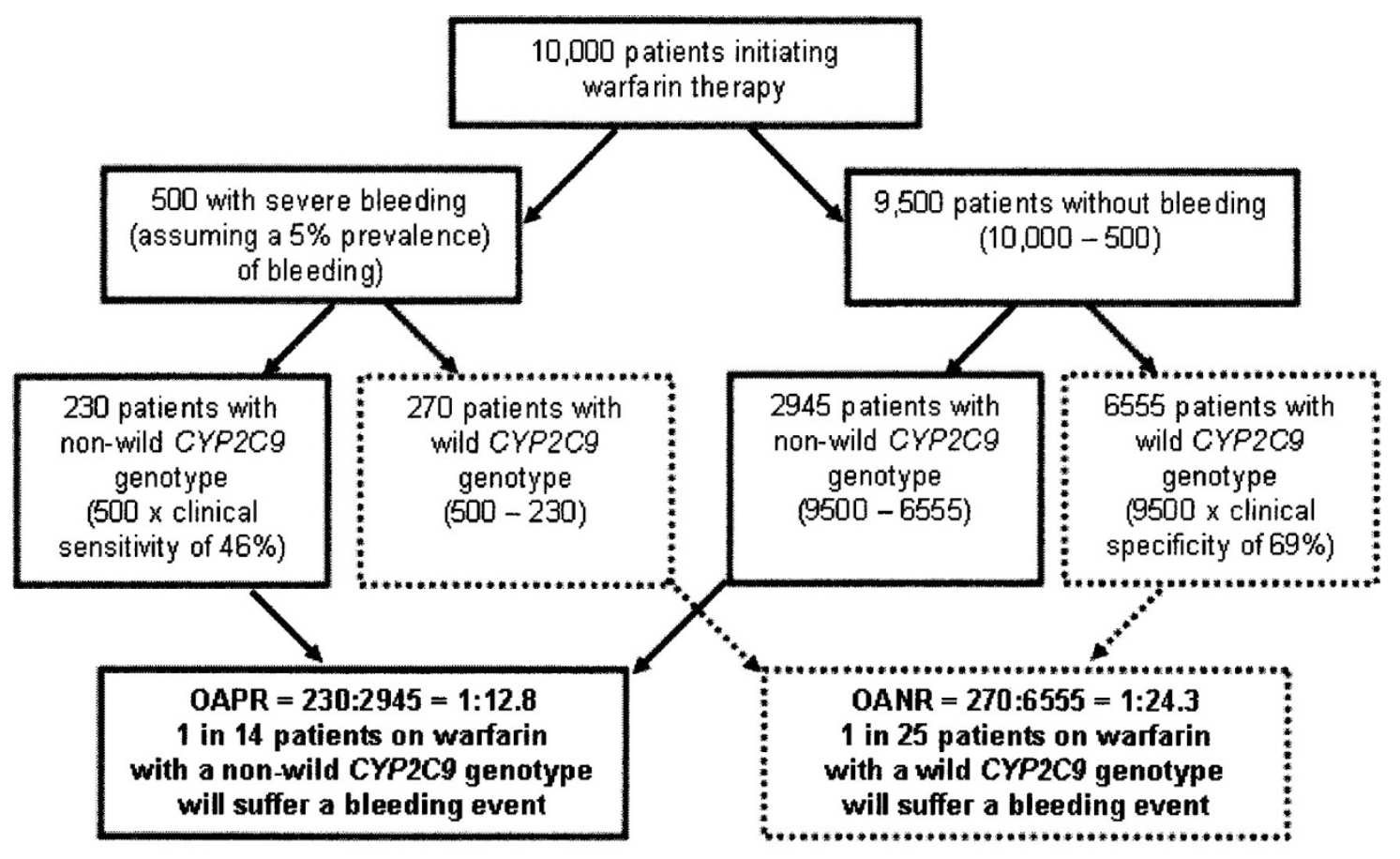

OAPR = odds of being affected given a positive result (non-wild genotype)

OANR = odds of being affected given a negative result (wild genotype)

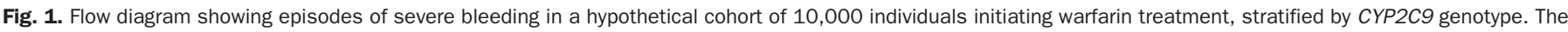

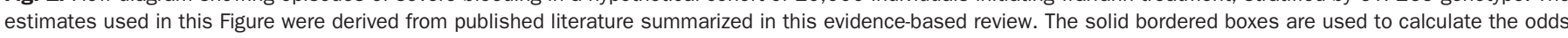

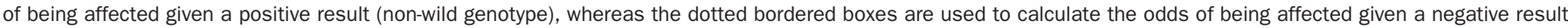
(wild genotype). 


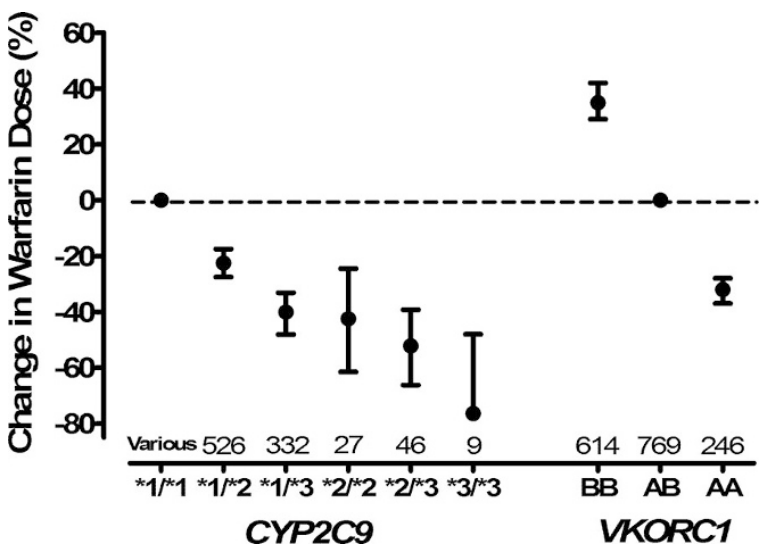

Fig. 2. Change in warfarin dose at stable INR by CYP2C9 or VKORC1 genotype. This meta-analysis includes 10 datasets for CYP2C9 genotyping and seven datasets for VKORC1 genotyping. The referent categories (horizontal dotted line) were chosen because they included the largest proportion of the population for each gene. The numbers above each genotype indicate the number of samples included in the analysis. For the CYP2C9 reference category, the number varied from a high of 1757 for the comparison with the $* 1 / * 3$ genotype to a low of 476 for the $* 3 / * 3$ genotype comparison.

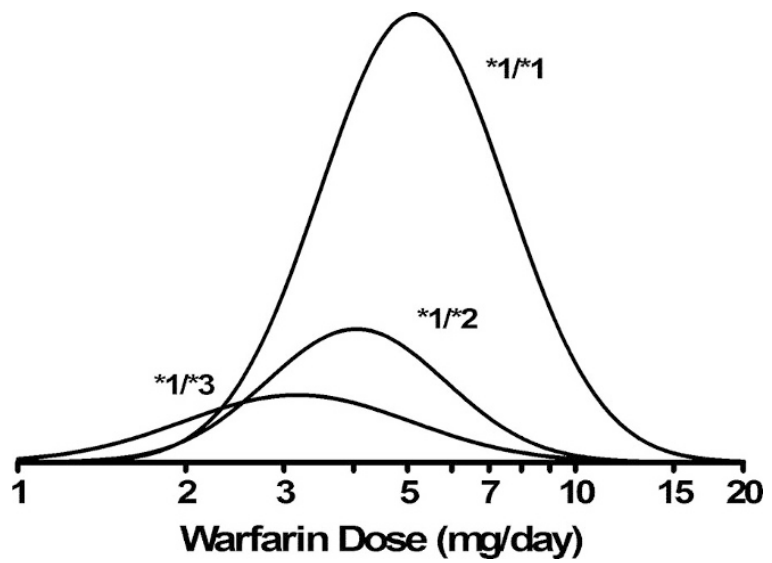

Fig. 3. Overlapping distributions of warfarin dose at stable INR for three CYP2C9 genotypes. The modeled distributions of warfarin dose are shown on a logarithmic horizontal axis. The areas of the three distributions are in direct relation to their prevalence $(* 1 / * 1$ being the most common). Although the reduction in stable warfarin dose is clearly visible for the $* 1 / * 2$ and $* 1 / * 3$ genotype, there is considerable overlap of the three distributions.

ence serious bleeding. Figure 1 also shows that the NPV is estimated to be $96 \%$ (i.e., 24 of 25 patients with a wild CYP2C9 genotype will not suffer a bleeding event).

Although not considered a direct measure of clinical validity, CYP2C9 genotypes are strongly related to warfarin dose, once the INR has stabilized. Compared with the wild genotype $\left({ }^{*} 1 /{ }^{*} 1\right)$, warfarin dose is reduced by $22 \%, 36 \%, 43 \%, 53 \%$, and $76 \%$ among individuals with the ${ }^{\star} 1 /{ }^{\star} 2,{ }^{*} 1 /{ }^{*} 3,{ }^{*} 2 /{ }^{*} 2,{ }^{\star} 2 /{ }^{*} 3$, and $\star 3 /{ }^{*}$ genotypes, respectively, (Fig. 2). ${ }^{11,12,22,31,32,47-52}$ Compared with the heterozygote VKORC1 genotype (indicated by $\mathrm{AB})$, warfarin dose is increased by $35 \%$ among individuals with the $\mathrm{BB}$ genotype and reduced by $32 \%$ among those with an $\mathrm{AA}$ genotypes. ${ }^{9-13,53}$ Figure 3 displays modeled distributions of stable warfarin dose for the three most common $C Y P 2 C 9$ genotypes, derived using data from one study. ${ }^{22}$ Although there

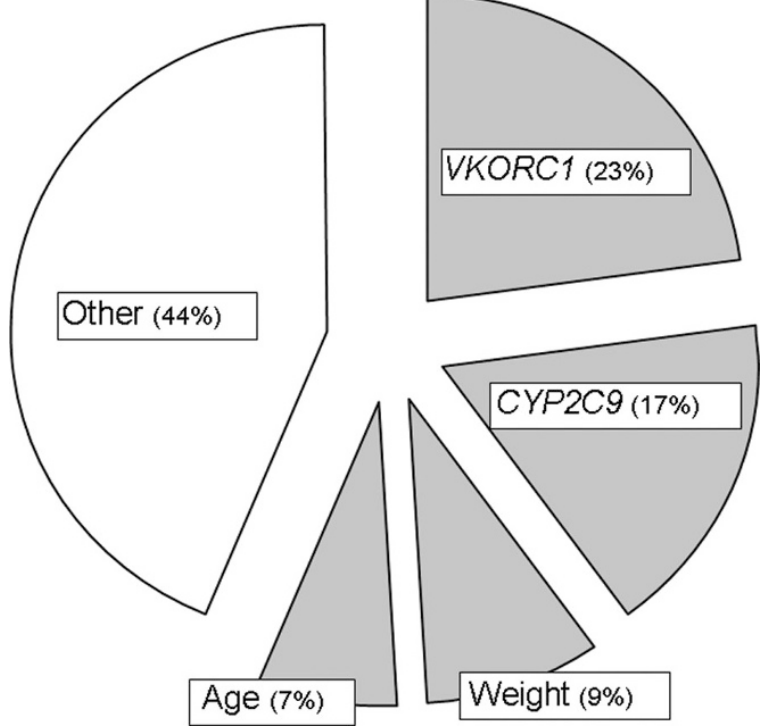

Fig. 4. Pie chart showing the known sources of variability in warfarin dose needed for a stable INR. Each estimate is based on a summary analysis of partial $r^{2}$ values from multivariate regression analysis reported in six studies that included genotyping on both CYP2C9 and VKORC1.

Table 5

Estimates of warfarin dose (mg) at stable INR, stratified by CYP2C9 and VKORC1 genotype

\begin{tabular}{|c|c|c|c|c|c|c|}
\hline \multirow{3}{*}{$\begin{array}{l}\text { VKORC1 } \\
\text { genotype }\end{array}$} & \multicolumn{6}{|c|}{ CYP2C9 genotype } \\
\hline & \multirow{2}{*}{$\begin{array}{l}\text { Rapid } \\
{ }^{*} 1 /{ }^{*} 1\end{array}$} & \multirow{2}{*}{$\begin{array}{l}\text { Inter } \\
{ }^{*} 1 /{ }^{*} 2\end{array}$} & \multicolumn{4}{|c|}{ Poor } \\
\hline & & & ${ }^{\star} 1 /{ }^{*} 3$ & $\star 2 / * 2$ & $* 2 / * 3$ & ${ }^{*} 3 /{ }^{*}$ \\
\hline High (BB) & 6.7 & 5.4 & 4.5 & 4.4 & 3.6 & 3.0 \\
\hline Medium (AB) & 4.8 & 3.9 & 3.2 & 3.2 & 2.6 & 2.2 \\
\hline Low (AA) & 3.5 & 2.8 & 2.3 & 2.3 & 1.9 & 1.6 \\
\hline
\end{tabular}

From www.WarfarinDosing.org, for a 65 -year-old Caucasian non-Hispanic man with a body surface area of $1.96 \mathrm{~m}^{2}$ (weight $=180 \mathrm{lbs}$, height $=5$ ' 8 ") with an initial INR of 0.75 and a target INR of 2.75 . He is a nonsmoker with no liver disease and is taking no relevant drugs (e.g., amiodarone, statin). The indication for warfarin is atrial fibrillation.

are clear reductions in the average levels, there is considerable overlap among these three groups. The three VKORC1 genotypes also have considerable overlap of stable warfarin dose (similar to Figure 10b in full report). CYP2C9 and VKORC1 genotypes contribute relatively independent information about stable warfarin dose (Fig. 4). ${ }^{10,12}$ Based on six studies that involved testing for both genes in a population with a steady state INR, VKORC1 haplotyping explains a slightly higher proportion of overall variability in warfarin dose (23\%) than CYP2C9 genotyping (17\%). ${ }^{9-13,53}$ This is because the VKORC1 genotypes associated with changes in dosage are more common in the Caucasian population. Other important factors in predicting warfarin dose are body weight (9\% of variability) and age ( $7 \%$ of variability). Four dosing models have been published, ${ }^{8,10,12,13}$ but none include both an appropriate transformation for warfarin dose (e.g., logarithmic) and allow for observed difference in warfarin doses for the ${ }^{\star} 1 /{ }^{\star} 2 \mathrm{vs}$. 
Table 6

Relative adjustments to warfarin dose at stable INR, stratified by CYP2C9 and VKORC1 genotype and estimated frequency per 1000

\begin{tabular}{|c|c|c|c|c|c|c|c|}
\hline \multirow[b]{3}{*}{ VKORC1 genotype } & \multicolumn{6}{|c|}{ CYP2C9 genotype } & \multirow[b]{3}{*}{ Frequency per 1000} \\
\hline & \multirow[b]{2}{*}{$\operatorname{Rapid}^{*} 1 /{ }^{\star} 1$} & \multirow[b]{2}{*}{ Inter ${ }^{\star} 1 /{ }^{\star} 2$} & \multicolumn{4}{|c|}{ Poor } & \\
\hline & & & ${ }^{*} 1 / * 3$ & $\star 2 / * 2$ & $* 2 / * 3$ & $* 3 / * 3$ & \\
\hline \multirow[t]{2}{*}{ High (BB) } & $140 \%$ & $113 \%$ & $94 \%$ & $92 \%$ & $75 \%$ & $63 \%$ & $(350)$ \\
\hline & $(223)$ & $(68)$ & $(44)$ & (5) & (7) & (2) & \\
\hline \multirow[t]{2}{*}{ Medium (AB) } & $100 \%$ & $81 \%$ & $67 \%$ & $67 \%$ & $54 \%$ & $46 \%$ & $(470)$ \\
\hline & $(300)$ & $(92)$ & (59) & (7) & (9) & (3) & \\
\hline \multirow[t]{2}{*}{ Low (AA) } & $73 \%$ & $58 \%$ & $48 \%$ & $48 \%$ & $40 \%$ & $33 \%$ & $(180)$ \\
\hline & $(115)$ & $(35)$ & $(23)$ & (3) & (3) & (1) & \\
\hline Frequency per 1000 & (638) & (195) & (126) & (15) & (19) & (6) & (1000) \\
\hline
\end{tabular}

The bolded entries $\left({ }^{*} 1 /{ }^{\star} 1 ; \mathrm{AB}\right)$ are the most common combination of CYP2C9/VKORC1 genotypes $(300 / 1000)$ and are considered the referent group (100\% dose). Other entries are represented as a percentage of this dose (e.g., $140 \%$ indicates a $40 \%$ increase in predicted dose to achieve a stable INR).

Frequencies are derived from the allele frequencies for CYP2C 9 of $12.2 \%$ and $7.9 \%$ for ${ }^{\star} 2$ and ${ }^{\star} 3$, respectively, and for the BB, $\mathrm{AB}$, and $\mathrm{AA}$ genotype frequencies of $35 \%, 47 \%$, and $18 \%$, respectively. The two sets of allele frequencies are considered to be independent.

From www.warfarindosing.org, for a 65-year-old Caucasian with a body surface area of $1.96 \mathrm{~m}^{2}$ (weight $=180 \mathrm{lbs}$, height $=5$ ' 8 ") with a target INR of 2.75 , who is a nonsmoker and is taking no other relevant drugs.

${ }^{\star} 1 /{ }^{\star} 3$ genotypes. Table 5 shows a comprehensive (but unpublished) warfarin dosing model (www.WarfarinDosing.org) that accounts for both CYP2C9 genotyping and VKORC1 genotyping, as well as several other known covariates. Since the completion of our review, two additional studies have been published that provide warfarin dosing models incorporating both CYP2C9 and VKORC1 variants. ${ }^{54,55}$ Both of these models include logarithmic transformation for warfarin dose and both CYP2C $9{ }^{\star} 2$ and ${ }^{*} 3$ genotypes. In addition, one of these models includes early warfarin doses and INR values. ${ }^{54}$ Table 6 shows these same warfarin doses relative to the most common subgroup $\left(\right.$ CYP2C9 $={ }^{\star} 1 /{ }^{\star} 1$, and $\left.V K O R C 1=\mathrm{AB}\right)$ comprising $30 \%$ of the Caucasian population. The display highlights that individuals with certain genotypes will actually receive a higher warfarin dose (e.g., $40 \%$ higher dose in $\left.{ }^{\star} 1{ }^{\star} 1, \mathrm{BB}\right)$, compared to those with the most common genotype.

Gaps in knowledge include: (1) the clinical sensitivity, clinical specificity, relative risk, and attributable risk of severe bleeding in the VKORC1 genotypes and in CYP2C9 and VKORC1 genotypes combined, (2) the contribution of genetic versus other influences toward bleeding in various racial/ethnic populations, (3) PPV and NPV for severe bleeding in the VKORC1 genotypes and CYP2C9 and VKORC1 genotypes combined, (4) how the difference in dosage would be best presented to clinicians who are initiating treatment in warfarin naïve individuals to ensure that a targeted dose will account for all known important sources of variation, and (5) the roles of other genes in the pharmacokinetics and pharmacodynamics of warfarin and their impact on warfarin dosage requirements.

\section{Clinical utility}

Clinical utility is defined as the benefits and risks associated with the introduction of a test into clinical practice, and includes economic analyses to determine the financial impact of such testing. This section begins by discussing the natural history of the disorder (severe bleeding).

Warfarin anticoagulation must be sufficient to avoid thrombotic events. However, excessive anticoagulation can result in severe, possibly fatal, bleeding events. The therapeutic window is narrow, and therapy is monitored by the international normalized ratio (INR), which is a standardized measure of the patient's prothrombin time, such that results are comparable across laboratories and test reagents. ${ }^{2}$ The target INR depends on the indication for anticoagulation, but the range is $2.0-3.0$ for most patients. INR monitoring usually begins $2-3$ days after the initial dose. ${ }^{56}$ In an acute, hospital setting, patients may be monitored daily; in an outpatient setting, two to three times weekly is recommended. If the INR remains stable, the interval can be gradually increased up to every 4 weeks. A steady state is usually achieved in 6-12 days (affected by CYP2C9 variants). ${ }^{7}$ If a $C Y P 2 C 9{ }^{*} 3$ variant is involved, the time to reach steady state may be two to three times longer than the expected 3-5 days in wild-type individuals.

The goal of long-term anticoagulation monitoring is to maintain the patient in the INR target range; success is measured as percent time in the therapeutic range and avoidance of adverse events. The stability of therapy over time may be influenced by changes in concomitant medications (including over-the-counter medications and nutraceuticals), health status changes that affect warfarin metabolism or vitamin K-dependent coagulation factors, dietary or gastrointestinal factors affecting vitamin $\mathrm{K}$ (e.g., alcohol use, irregular ingestion of vitamin K-rich foods, changes in intestinal absorption capacity). It is important that the health care provider monitor at appropriate intervals, consider any changes in status, and make necessary and appropriate dose adjustments to maintain INR in the target range. In addition, patient communication, education, and compliance are important determinants of success. 
Finally, active intervention may be required when the INR is excessively prolonged and the patient has active bleeding or is at high risk for bleeding.

The intended action of CYP2C9 and VKORC1 alleles testing is to predict an individual's maintenance warfarin dose by incorporating demographic, clinical, and gene variant data (both CYP2C9 and VKORC1). This can be used as the initial dose to limit high INR values (over-anticoagulation) that are associated with serious bleeding events, and to decrease time to stable INR. Many of these events will occur within the first few weeks of treatment. No study has yet shown this intervention to be effective in reducing the incidence of high INR values, the time to stable INR, or the occurrence of serious bleeding events. One small pilot randomized trial enrolled 38 patients and found six serious bleeding events among the 20 patients with standard warfarin dosing versus two bleeding events among the 18 receiving model-based dosing using CYP2C9 genotyping. ${ }^{57}$ These results are not statistically significant, but show acceptability of the randomized design. Several large randomized trials are underway to determine the clinical effectiveness of CYP2C9 genotyping and VKORC1 haplotyping to inform warfarin dosing. Some of these trials are using severe bleeding as the outcome, whereas others are targeting intermediate measures such as reducing the time to achieve stable INR, and the percentage of time in range during dose stabilization.

Using estimates of clinical validity described earlier (Fig. 1), along with several assumptions of clinical utility (e.g., cost of testing and the effectiveness of targeted warfarin dose to avoid serious bleeding), the number of individuals that must be tested to avoid one serious bleeding event ranges from 48 to 385 . The cost per serious bleeding event averted ranges from $\$ 14,500$ to $\$ 95,900$. Key assumptions that strongly influence this cost estimate are the effectiveness of targeted warfarin dose (range $80-20 \%$ in a sensitivity analysis) and the cost of genetic testing (range $\$ 300-\$ 500$ ).

Economic outcomes and decision analysis studies on genetic and pharmacogenetic testing have been published. ${ }^{58-60}$ One recently released analysis suggests that genetic testing before warfarin dosing will avoid many severe bleeding events and result in large cost savings. ${ }^{60}$ However, close examination of this study reveals that the authors made several assumptions that may not be valid. These include: targeted dosing by genotype will be $100 \%$ effective in reducing bleeding events to the level of that in individuals with the wild genotype, more effective dosing will reduce the rate of strokes, a rate of bleeding events that is higher than expected, and a relatively high estimate of new warfarin users per year.

Gaps in knowledge include: (1) the clinical utility of genotyping before warfarin dosing (e.g., is there a reduction in time to stable INR, is there a reduction in severe bleeding events?), (2) cost-effectiveness of VKORC1 testing alone, or in combination with CYP2C9, (3) the impact of the timing of genotyping (e.g., before initial dose or 2-3 days after initial warfarin treatment), (4) validated educational materials for patients and providers, (5) long-term monitoring plans, and (6) guidelines for evaluating program performance.

\section{Ethical, legal, and social implications (ELSI)}

Pharmacogenomic testing might be perceived as carrying less serious ELSI than other types of genetic testing. For example, a variant that alters response to a drug (e.g., a CYP2C9 or VKORC1 genotype) might carry less potential for discrimination, privacy/confidentiality, and stigmatization than a mutation that is predictive of a debilitating and/or fatal disease (e.g., Huntington disease). However, a premise does exist that pharmacogenomic tests may be used to classify groups that face discrimination in health care, resulting in prejudice and stigmatization..$^{30,61}$ Furthermore, stratifying the population into genetic subgroups could mean that the costs of developing new drugs tailored to the needs of a given small subgroup might be prohibitively expensive and might not be developed. Even if this premise should bear out, an individual will still receive benefit, if found to be in a genetic subgroup for which an existing therapy is known to be harmful, in that inappropriate treatment will be avoided.

The Nuffield Council on Bioethics Report ${ }^{62}$ suggests that "the likelihood that pharmacogenomic data will be of relevance to family members is low." Although single nucleotide polymorphisms (SNPs) are heritable, SNP testing has not been widely studied, and it may be too early to decide definitively whether this statement will be upheld.

Pharmacogenetic testing for CYP2D6, in the context of tamoxifen use, is already being marketed directly to consumers (www.DNAdirect.com). Stand-alone CYP2D6 testing for generalized drug metabolism is advertised, but not yet available. The issues of direct-to-consumer marketing of genetic tests have been discussed elsewhere. ${ }^{63,64}$ It is likely that $C Y P 2 C 9$ and $V K O R C 1$ testing will also be offered directly to consumers in the near future.

It has been recommended that, if information about unrelated medicines or diseases is likely to be obtained from pharmacogenomic testing, or if the results of the test will have a significant impact on the health or lifestyle of the patient, written consent may be appropriate. ${ }^{62}$ Even if it is decided that consent is not required, written information (e.g., education materials) might be appropriate.

Legal implications may arise as pharmacogenomic testing becomes widespread. For instance, will providers and drug companies be held liable for not considering genetic information? Should pharmacies store genotype information obtained for one application and use it when dispensing other drugs utilizing the same metabolic pathway? Finally, the new FDArevised warfarin label may make the conduct of randomized controlled trials more difficult.

The issues discussed in this section are all considered gaps in knowledge and will require further monitoring and documentation to further describe the ethical, legal, and social implications of pharmacogenomic testing.

\section{DISCUSSION}

Justifications for performing a Rapid-ACCE review at this time include: the potential for a large number of genetic tests to 
be performed (as many as 1 or 2 million new warfarin patients per year), the high rate of adverse drug events associated with warfarin usage (800/year in the United States), the FDA-revised Coumadin ${ }^{\circledR}$ label that includes genomic test information, and the availability of CYP2C9/VKORC1 testing services. The structure of the rapid-ACCE evidence review can be applied to other emerging tests. The objectives of this review were to: (1) briefly evaluate and summarize existing knowledge, (2) provide information to aid in developing clinical and laboratory guidelines for CYP2C9 and VKORC1 alleles testing to guide warfarin dosing, (3) provide information to be used in provider and patient education materials, and (4) identify gaps in knowledge from which a research agenda can be developed.

We found data showing the analytic validity of CYP2C9 allele testing to be acceptable, but data are lacking for that of VKORC1 allele testing. Results from the new College of American Pathology proficiency testing program will improve the evidence base. There exists compelling evidence for the association between CYP2C9 and VKORC1 genotypes and stable warfarin dose. Fewer data are available to evaluate the association between CYP2C9 genotype and stable INR during the induction phase, when the risk of severe bleeding is highest. There are very limited data on the clinical validity of CYP2C9 genotyping to predict severe bleeding events, and no data for VKORC1 genotypes. The clinical utility of DNA testing in this clinical scenario is to "personalize" an individual's initial warfarin dose by incorporating demographic, clinical, and genotype data (CYP2C9 and VKORC1), as a way to limit high INR values (over-anticoagulation) that are associated with an increased risk of serious bleeding events. No large study has yet shown this to be acceptable or effective. Several randomized trials are underway to determine the clinical effectiveness of CYP2C9 and VKORC1 genotyping to inform warfarin dosing to reduce serious bleeding. There are several ethical, legal, and social implications that need to be monitored to ensure equitable, nondiscriminatory, and confidential CYP2C9 and VKORC1 testing.

\section{ACKNOWLEDGMENTS}

Funding was provided by the American College of Medical Genetics Foundation (ACMGF). Tm Bioscience provided the ACMGF with partial funding for this study.

We thank David Flockhart, MD, PhD, Indiana University School of Medicine, Indianapolis, Indiana; Dennis O'Kane, $\mathrm{PhD}$, Mayo Clinic, Rochester, Minnesota; Michael Watson, $\mathrm{PhD}$, American College of Medical Genetics, Washington, DC; and Marc S. Williams, MD, Clinical Genetics Institute, LDS Hospital, Salt Lake City, Utah, for their oversight and comments.

We also thank Amy Brower, PhD, Third Wave Technologies, Madison, Wisconsin; LabCorp, Burlington, North Carolina; Brian Gage, MD, Washington University School of Medicine, St. Louis, Missouri; Roy Gandolfi, MD, Intermountain Health care, West Valley City, Utah; Elaine Lyon, PhD, ARUP, Salt Lake City, Utah; David Veenstra, PharmD, University of Washington, Seattle, Washington; and Ann Wittkowsky,
PharmD, University of Washington Medical Center, Seattle, Washington, for their submission of unpublished data, discussions and/or comments.

\section{References}

1. Haddow JE, Palomaki GE. ACCE: a model process for evaluating data on emerging genetic tests. In: Khoury MJ, Little J, Burke W, editors. Human genome epidemiology: a scientific foundation for using genetic information to improve health and prevent disease. Oxford: Oxford University Press, 2003.

2 Johnston M, Harrison L, Moffat K, Willan A, et al. Reliability of the international normalized ratio for monitoring the induction phase of warfarin: comparison with the prothrombin time ratio. J Lab Clin Med 1996;128:214 -217.

3. Moore TJ, Cohen MR, Furberg CD. Serious adverse drug events reported to the Food and Drug Administration, 1998-2005. Arch Intern Med 2007;167:1752-1759.

4. Gudgeon JM, McClain MR, Palomaki GE, Williams MS. Rapid ACCE: experience with a rapid and structured approach for evaluating gene-based testing. Genet Med 2007;9:473-478.

5. Flockhart DA, O’Kane D, Williams MS, Watson MS, et al. Pharmacogenetic testing of CYP2C9 and VKORC1 alleles for warfarin. Genet Med 2008;10:139-150.

6. Sanderson S, Emery J, Higgins J. CYP2C9 gene variants, drug dose, and bleeding risk in warfarin-treated patients: a HuGEnet systematic review and meta-analysis. Genet Med 2005;7:97-104.

7. Linder MW, Looney S, Adams JE III, Johnson N, et al. Warfarin dose adjustments based on CYP2C9 genetic polymorphisms. J Thromb Thrombolysis 2002;14:227232 .

8. Takahashi H, Wilkinson GR, Nutescu EA, Morita T, et al. Different contributions of polymorphisms in VKORC1 and CYP2C9 to intra- and inter-population differences in maintenance dose of warfarin in Japanese, Caucasians and African-Americans. Pharmacogenet Genomics 2006;16:101-110.

9. Rieder MJ, Reiner AP, Gage BF, Nickerson DA, et al. Effect of VKORC1 haplotypes on transcriptional regulation and warfarin dose. N Engl J Med 2005;352:2285-2293.

10. Aquilante C L, Langaee TY, Lopez LM, Yarandi NH, et al. Influence of coagulation factor, vitamin $\mathrm{K}$ epoxide reductase complex subunit 1, and cytochrome P450 2C9 gene polymorphisms on warfarin dose requirements. Clin Pharmacol Ther 2006;79: 291-302.

11. D'Andrea G, D'Ambrosio RL, Di Perna P, Chetta M, et al. A polymorphism in the VKORC1 gene is associated with an interindividual variability in the dose-anticoagulant effect of warfarin. Blood 2005;105:645-649.

12. Sconce EA, Khan TI, Wynne HA, Avery P, et al. The impact of CYP2C9 and VKORC1 genetic polymorphism and patient characteristics upon warfarin dose requirements: proposal for a new dosing regimen. Blood 2005;106:2329 -2333.

13. Vecsler M, Loebstein R, Almog S, Kurnik D, et al. Combined genetic profiles of components and regulators of the vitamin K-dependent gamma-carboxylation system affect individual sensitivity to warfarin. Thromb Haemost 2006;95:205-211.

14. Geisen C, Watzka M, Sittinger K, Steffens M, et al. VKORC1 haplotypes and their impact on the inter-individual and inter-ethnical variability of oral anticoagulation. Thromb Haemost 2005;94:773-779.

15. Lee SC, Ng SS, Oldenburg J, Chong PY, et al. Interethnic variability of warfarin maintenance requirement is explained by VKORC1 genotype in an Asian population. Clin Pharmacol Ther 2006;79:197-205.

16. Li T, Lange L, Li X, Susswein L, et al. Polymorphisms in the VKORC1 gene are strongly associated with warfarin dosage requirements in patients receiving anticoagulation. J Med Genet 2006;43:740 -744.

17. Mushiroda T, Ohnishi Y, Saito S, Takahashi A, et al. Association of VKORC1 and CYP2C9 polymorphisms with warfarin dose requirements in Japanese patients. J Hum Genet 2006;51:249 -253.

18. Veenstra DL, You JH, Rieder MJ, Farin FM, et al. Association of vitamin K epoxide reductase complex 1 (VKORC1) variants with warfarin dose in a Hong Kong Chinese patient population. Pharmacogenet Genomics 2005;15:687- 691.

19. Aquilante CL, Lobmeyer MT, Langaee TY, Johnson JA. Comparison of cytochrome P450 2C9 genotyping methods and implications for the clinical laboratory. Pharmacotherapy 2004;24:720 -726.

20. Burian M, Grosch S, Tegeder I, Geisslinger G. Validation of a new fluorogenic real-time PCR assay for detection of CYP2C9 allelic variants and CYP2C9 allelic distribution in a German population. Br J Clin Pharmacol 2002;54:518 -521.

21. Eriksson S, Berg LM, Wadelius M, Alderborn A. Cytochrome p450 genotyping by multiplexed real-time DNA sequencing with pyrosequencing technology. Assay Drug Dev Technol 2002;1(1, Pt 1):49 -59.

22. Hillman MA, Wilke RA, Caldwell MD, Berg RL, et al. Relative impact of covariates in prescribing warfarin according to CYP2C9 genotype. Pharmacogenetics 2004;14: 539-547.

23. Pickering JW, McMillin GA, Gedge F, Hill HR, et al. Flow cytometric assay for 
genotyping cytochrome p450 2C9 and 2C19: comparison with a microelectronic DNA array. Am J Pharmacogenomics 2004;4:199 -207.

24. Wen SY, Wang H, Sun OJ, Wang SQ. Rapid detection of the known SNPs of CYP2C9 using oligonucleotide microarray. World J Gastroenterol 2003;9:1342-1346.

25. Zainuddin Z, Teh LK, Suhaimi AW, Salleh MZ, et al. A simple method for the detection of CYP2C9 polymorphisms: nested allele-specific multiplex polymerase chain reaction. Clin Chim Acta 2003;336:97-102.

26. Palomaki GE, Bradley LA, Richards CS, Haddow JE. Analytic validity of cystic fibrosis testing: a preliminary estimate. Genet Med 2003;5:15-20.

27. Palomaki GE, Haddow JE, Bradley LA, Richards CS, et al. Estimated analytic validity of HFE C282Y mutation testing in population screening: the potential value of confirmatory testing. Genet Med 2003;5:440-443.

28. Stuhrmann M, Strassburg C, Schmidtke J. Genotype-based screening for hereditary haemochromatosis. I. Technical performance, costs and clinical relevance of a German pilot study. Eur J Hum Genet 2005;13:69 -78.

29. Hruska MW, Frye RF, Langaee TY. Pyrosequencing method for genotyping cytochrome P450 CYP2C8 and CYP2C9 enzymes. Clin Chem 2004;50:2392-2395.

30. Ogg MS, Brennan P, Meade T, Humphries SE. CYP2C $9 * 3$ allelic variant and bleeding complications. Lancet 1999;354:1124.

31. Margaglione M, Colaizzo D, D'Andrea G, Brancaccio V, et al. Genetic modulation of oral anticoagulation with warfarin. Thromb Haemost 2000;84:775-778.

32. Higashi MK, Veenstra DL, Kondo LM, Wittkowsky AK, et al. Association between CYP2C9 genetic variants and anticoagulation-related outcomes during warfarin therapy. JAMA 2002;287:1690 - 1698 .

33. Wadelius M, Sorlin K, Wallerman O, Karlsson J, et al. Warfarin sensitivity related to CYP2C9, CYP3A5, ABCB1 (MDR1) and other factors. Pharmacogenomics J 2004;4: $40-48$.

34. Lindh JD, Lundgren S, Holm L, Alfredsson L, et al. Several-fold increase in risk of overanticoagulation by CYP2C9 mutations. Clin Pharmacol Ther 2005;78:540 -550.

35. Peyvandi F, Spreafico M, Siboni SM, Moia M, et al. CYP2C9 genotypes and dose requirements during the induction phase of oral anticoagulant therapy. Clin Pharmacol Ther 2004;75:198 -203.

36. Wilke RA, Berg RL, Vidaillet HJ, Caldwell MD, et al. Impact of age, CYP2C9 genotype and concomitant medication on the rate of rise for prothrombin time during the first 30 days of warfarin therapy. Clin Med Res 2005;3:207-213.

37. Enyart JJ, Jones RJ. Low-dose warfarin for prevention of symptomatic thromboembolism after orthopedic surgery. Ann Pharmacother 2005;39:1002-1007.

38. Fitzgerald RH Jr, Spiro TE, Trowbridge AA, Gardiner GA Jr, et al. Prevention of venous thromboembolic disease following primary total knee arthroplasty. A randomized, multicenter, open-label, parallel-group comparison of enoxaparin and warfarin. J Bone Joint Surg Am 2001;83:900 -906.

39. Francis CW, Berkowitz SD, Comp PC, Lieberman JR, et al. Comparison of ximelagatran with warfarin for the prevention of venous thromboembolism after total knee replacement. N Engl J Med 2003;349:1703-1712.

40. Freedman KB, Brookenthal KR, Fitzgerald RH Jr, Williams S, et al. A meta-analysis of thromboembolic prophylaxis following elective total hip arthroplasty. J Bone Joint Surg Am 2000;82:929 -938.

41. Hull RD, Pineo GF, Francis C, Bergqvist D, et al. Low-molecular-weight heparin prophylaxis using dalteparin in close proximity to surgery vs warfarin in hip arthroplasty patients: a double-blind, randomized comparison. The North American Fragmin Trial Investigators. Arch Intern Med 2000;160:2199 -2207.

42. Levine MN, Raskob G, Beyth RJ, Kearon C, et al. Hemorrhagic complications of anticoagulant treatment: the Seventh ACCP Conference on Antithrombotic and Thrombolytic Therapy. Chest 2004;126(3 suppl):287S-310S.

43. Prandoni $\mathrm{P}$, Bruchi O, Sabbion $\mathrm{P}$, Tanduo C, et al. Prolonged thromboprophylaxis with oral anticoagulants after total hip arthroplasty: a prospective controlled randomized study. Arch Intern Med 2002;162:1966 -1971.

44. Sachs RA, Smith JH, Kuney M, Paxton L. Does anticoagulation do more harm than good? A comparison of patients treated without prophylaxis and patients treated with low-dose warfarin after total knee arthroplasty. J Arthroplasty 2003;18:389395.

45. Stern SH, Wixson RL, O'Connor D. Evaluation of the safety and efficacy of enoxaparin and warfarin for prevention of deep vein thrombosis after total knee arthroplasty. J Arthroplasty 2000;15:153-158.

46. Vives MJ, Hozack WJ, Sharkey PF, Moriarty L, et al. Fixed minidose versus-adjusted low-dose warfarin after total joint arthroplasty: a randomized prospective study. J Arthroplasty 2001;16:1030 -1037.

47. Joffe HV, Xu R, Johnson FB, Longtine J, et al. Warfarin dosing and cytochrome P450 2C9 polymorphisms. Thromb Haemost 2004;91:1123-1128.

48. Loebstein R, Yonath H, Peleg D, Almog S, et al. Interindividual variability in sensitivity to warfarin—nature or nurture? Clin Pharmacol Ther 2001;70:159-164.

49. Scordo MG, Pengo V, Spina E, Dahl ML, et al. Influence of CYP2C9 and CYP2C19 genetic polymorphisms on warfarin maintenance dose and metabolic clearance. Clin Pharmacol Ther 2002;72:702-710.

50. Tabrizi AR, Zehnbauer BA, Borecki IB, McGrath SD, et al. The frequency and effects of cytochrome $\mathrm{P} 450$ (CYP) $2 \mathrm{C} 9$ polymorphisms in patients receiving warfarin. J Am Coll Surg 2002;194:267-273.

51. Taube J, Halsall D, Baglin T. Influence of cytochrome P-450 CYP2C9 polymorphisms on warfarin sensitivity and risk of over-anticoagulation in patients on longterm treatment. Blood 2000;96:1816 -1819.

52. Topic E, Stefanovic M, Samardzija M. Association between the CYP2C9 polymorphism and the drug metabolism phenotype. Clin Chem Lab Med 2004;42:72-78.

53. Wadelius M, Chen LY, Downes K, Ghori J, et al. Common VKORC1 and GGCX polymorphisms associated with warfarin dose. Pharmacogenomics J 2005;5:262-270.

54. Millican E, Jacobsen-Lenzini PA, Milligan PE, Grosso L, et al. Genetic-based dosing in orthopaedic patients beginning warfarin therapy. Blood 2007;110:1511-1515.

55. Zhu Y, Shennan M, Reynolds KK, Johnson NA, et al. Estimation of warfarin maintenance dose based on VKORC1 ( $1639 \mathrm{G}$ A) and CYP2C9 genotypes. Clin Chem 2007;53:1199-1205.

56. Hirsh J, Guyatt GH, Albers GW, Schunemann HJ. The seventh ACCP conference on antithrombotic and thrombolytic therapy: evidence-based guidelines. Chest 2004; 126:172S-173S.

57. Hillman MA, Wilke RA, Yale SH, Vidaillet HJ, et al. A prospective, randomized pilot trial of model-based warfarin dose initiation using CYP2C9 genotype and clinical data. Clin Med Res 2005;3:137-145.

58. Higashi MK, Veenstra DL. Managed care in the genomics era: assessing the cost effectiveness of genetic tests. Am J Manag Care 2003;9:493-500.

59. You JH, Chan FW, Wong RS, Cheng G. The potential clinical and economic outcomes of pharmacogenetics-oriented management of warfarin therapy-a decision analysis. Thromb Haemost 2004;92:590-597.

60. McWilliam A, Lutter R, Nardinelli C. Health care savings from personalizing medicine using genetic testing: the case of warfarin. Working paper 06-23. Washington, DC: AEI-Brookings Joint Center for Regulatory Studies, 2006.

61. Schubert L. Ethical implications of pharmacogenetics- do slippery slope arguments atter? Bioethics 2004;18:361-378.

62. Parmacogenetics: ethical issues. London, UK: Nuffield Council on Bioethics, 2003.

63. Gollust SE, Hull SC, Wilfond BS. Limitations of direct-to-consumer advertising for clinical genetic testing. JAMA 2002;288:1762-1767.

64. Hull SC, Prasad K. Reading between the lines: direct-to-consumer advertising ofgenetic testing in the USA. Reprod Health Matters 2001;9:44-48. 